\title{
THE PROBLEMS OF STANDARDIZATION OF METHODS IN USE IN MICROBIOLOGICAL SOIL RESEARCH ${ }^{1}$ )
}

\author{
K. T. WIERINGA \\ Laboratory of Microbiology, Wageningen, Netherlands
}

A symposium organized by the International Association of Soil Science, Commission III - Soil Biology, was held at Louvain from 3 to 5 June 1957. The subject of this symposium was a discussion of the methods used in soil microbiology, the ultimate object being to tandardize the methods used in various laboratories. The subjects discussed were presented in a number of general reports, viz. :

1 the sampling of soils (Ziemiecka, Pologne),

2 the determination of biological activity (Mishoustine, U.R.S.S.),

3 the study and counting of soil bacteria (Pochon, France),

4 the study and counting of soil fungi (Verona, Italy and Simonart, Belgium), and

5 a report on the descriptive chart of standard methods used in the biological examination of the soil to be given by the present author.

I felt my task was rather an onerous one, especially as I believe that standardization in so complex a subject as soil microbiology is impracticable in view of our present knowledge. A great deal of research will have to be done before standard methods are acceptable.

The biological activity of the soil is substantially of an enzymatic kind, the enzymes being produced by the living microflora and fauna. Hence the numbers of micro-organisms and their activity determine the biological activity of the soil. The factors mentioned depend on the fertility of the soil. Soil fertility in its turn is a very complex conception. In its most simple form we may regard it as the property of the soil to produce a crop, the most fertile soil being that which produces the largest crop for the least outlay and labour.

The factors that determine fertility are numerous. We may mention, for example, the texture of the soil, which determines the interdependent rates of the 3 soil phases: solid substance, liquid, and gas, and which in turn depends on the soil colloids, both organic and inorganic.

The colloidal soil complex is at the same time a most important factor for the presence and availibility of plant nutrients; it controls the buffering capacity against acid and alkali and regulates the fluctuations in $\mathrm{pH}$. It is the task of the farmer to maintain a good texture. Good maintenace will do a great deal ; regular dressings of organic matter will nearly always be necessary. Abnormal maintenance will lead to abnormal composition of the microbiological activity in the soil.

For us this means that a descriptive chart should include standardized methods for estimating soil texture, water capacity, $\mathrm{pH}$ and buffering capacity.

1) Received for publication August 13, 1957. 
When organic matter is added to the soil the slow activity of WINOGRADSKY's (7) autochtonous flora is overlapped by the "wild flora" which is in a quiescent state when the soil is in a biological equilibrium, as is the case in the "terre témoin" untouched for a number of years. The flourishing of the wild flora, which WiNogransky terms "zymogène", depends on the nature of the substances added, but will also vary with the nature of the "terre témoin". The soil is an infusion in which one process takes place together with others, each being again succeeded by others. Dependent on soil conditions, mineralization will be the result in a more or less complete sense until the biological activity reverts to the starting point of the fallow, or "terre témoin".

WINOGRADSKY (7) was the first to emphasize the study of biological soil activity in the soil itself. He introduced the "culture spontanée" in order to study the synthesis of soil microbiology, and he urged us to drop the classic elective culture methods which are analytical. His warnings caused a great sensation in the world of soil microbiologists, especially as they were uttered by this great master of microbiology who had spent the first part of his scientific career on elective culture methods which were now rejected. Despite this we were unable to dispense with the knowledge acquired by these methods and are obliged to return to them constantly in our work.

This is also true of the much criticized plating method, although we realise its great drawbacks. I need not list all these, it will be sufficient to mention a few of them. It is quite impossible to choose a medium that will enable all micro-organisms to grow. The microbes are present in the soil in a discontinuous state, either as small colonies or threads. Shaking a soil suspension or treating it in some other way will break up these colonies or threads in an uncontrollable way. Other mistakes are made in preparing the dilution of the soil sample. For instance, a series of 6 dilutions is made. From the last of these dilutions a number of plates are inoculated. WAKSMAN (5) advised the use of 10 parallel plates in order to obtain results that allow mathematical treatment. Would it not then be logical to make 10 parallels in each dilution? But this would ultimately mean $10^{6}$ dilutions for one sample, in which case the method would be totally impracticable.

I hope to show by means of an example that the plate count can still give good results if there is sufficient difference between the soil samples compared.

In the middle of the thirties ploughing up of old grassland became a matter of urgency in Holland. I was interested to see what effect ploughing would have on the soil microflora. Soil samples taken from old grassland and arable land, both clay and sandy soil, were collected in the neighbourhood of Wageningen. All these samples were taken at a depth of 5-10 cm. Some samples originated from soils ploughed up in the years 1934, '35 and '36. As soon as the samples had been taken they were brought to the laboratory and suspensions were made containing the equivalent of $0.01 \mathrm{mg}$ of the soil sample per $0.5 \mathrm{ml}$. To avoid as far as possible the errors made in pipetting and diluting I made as few dilutions as possible. $1 \mathrm{~g}$ of soil was introduced in $1 \mathrm{l}$ of sterile tapwater, shaken by hand for 5 minutes and, while shaking was continued, $1 \mathrm{ml}$ of this suspension was introduced in $50 \mathrm{ml}$ sterile tapwater, $0.5 \mathrm{ml}$ of the latter suspension being plated on the surface of agar plates; 5 parallel plates were made. A duplicate gram of soil was treated in the same way. For each dilution a new sterile pipette was used. The soil suspensions were sucked 
up 5 times in the pipette in order to saturate all points of the inner wall of the pipette which were capable of absorbing micro-organisms.

The nutrient agar consisted of the extract of a fertile clay soil containing $1.5 \%$ pure washed agar, $0.5 \% \mathrm{Ca}$-caseinate and $0.1 \% \mathrm{~K}_{2} \mathrm{HPO}_{4}$. The casein was added as a saturated solution in $\mathrm{Ca}(\mathrm{OH})_{2}$-water. It is important for the plates poured from this agar to be dried before plating the $0.5 \mathrm{ml}$ suspension on them, so that they will take up the water readily. If the suspension is spread on the agar surface while the latter is lukewarm the water is taken up readily and spreading colonies will be largely avoided; it is only colonies of the mycoides type that may make enumeration impossible. Such plates should ne discarded. A considerable advantage of the cultivation on the surface of the agar plates is that under these conditions the types of micro-organisms can be recognized by the appearance of the colonies and divided into groups.

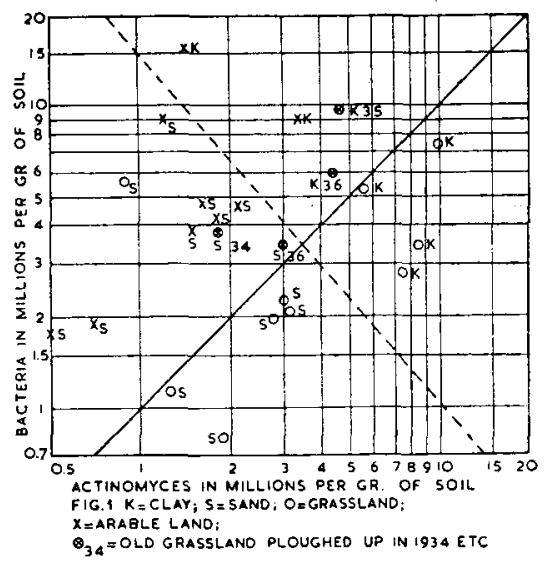

Fig. 1.

On these plates bacteria and actinomyces grow readily and are easily recognizable, so that after incubation for 4 or 5 days at $27^{\circ} \mathrm{C}$ the ratio of actinomyces (a) : bacteria (b) $=a / b$ can be determined without difficulty. Fig. 1 shows the result of the experiment including 23 soils, including 4 ploughed-up plots of old grassland. One line is drawn to divide the graph into two ; above this line $\mathrm{a}<\mathrm{b}$ and below $\mathrm{a}>\mathrm{b}$. A dotted line is drawn so as to leave the sandy soils on the left and the clay soils on the right. The graph shows that in all old grasslands except one the actinomyces are in excess of the bacteria. In arable soils the situation is the reverse. Furthermore, the clay soils belong to the types with high total numbers, whereas sandy soils show much smaller numbers. The effect of ploughing up is to reverse immediately the ratio $a / b$. All four ploughed-up soils belong to the type in which bacteria form the greater part of the population growing on the caseinate plates. Hence in this experiment the "biotype" was characteristic of the soil samples examined:

Arable sand .......... low numbers; $a<b$

Grassland sand $\ldots \ldots \ldots$ low numbers; $a>b$

Arable clay $\ldots \ldots \ldots \ldots$ high numbers $; a<b$

Grassland clay ......... high numbers; $a>b$ 
We may conclude that in arable land the dissimilation of organic matter is more rapid than in grassland, and this is a fair inference as every farmer knows that old grassland is a rich storehouse of organic matter.

The main point of this experiment was not the counting of total numbers of micro-organisms, although the ratio in which two types of micro-organisms developed on the caseinate plates was characteristic of the agricultural use of the soil. When plate counts are used for this purpose failures made in diluting and pipetting of the soil suspensions become less important as they do not seriously interfere with the ratio of micro-organisms present.

In this experiment all the samples were taken under the same climatic conditions and investigated immediately. Consequently I was not concerned with a number of difficult problems inherent in soil sampling. If we are standardizing the methods of estimating the biological activity of the soil, at what time of the year should we take the sample, - in spring, summer, autumn or winter? Should we take the sample after a period of drought or after rain has fallen? If the sample is taken, how long may we defer its examination? Should we keep our samples in the laboratory under standardized conditions and, if so, for how long? What would be the best moisture content and at what temperature should the sample be kept? As long as we are not clear on these points, standardizing of other microbiological methods is of minor importance.

Another important point is the standardization of the nutrient medium. If soil extract is taken as the basic medium, will it be possible to prepare a medium that gives comparable results in various laboratories?

I am sure there is a lot of work to be done before we can arrive at satisfactory standardized methods as far as these points are concerned.

Some of the difficulties mentioned above are met with in a group of methods for measuring $\mathrm{CO}_{2}$ production by the soil, viz. those inherent in soil sampling. If, however, only fresh samples are examined which have been brought to a moisture content of $60 \%$ of the water capacity, most of the difficulties are overcome. The production of $\mathrm{CO}_{2}$ is a biological activity involving all micro-organisms in the soil. There are many ways of measuring this activity which I will not summarise in this report. I will only make an exception in the case of one simple method, i.e. that introduced by Petersen (Lyngby) (3). A gauze bag containing the soil sample is suspended in an Erlenmeyer flask on the bottom of which is a certain amount of barytes. The flask is stoppered. After incubation for a number of days the $\mathrm{CO}_{2}$ formed and caught in the barytes is estimated. This method is very simple and in my opinion as good as any other. Actually the soil is not in its natural condition, but standardized conditions will make comparison possible.

Another point is the uptake of oxygen in respiration as compared with $\mathrm{CO}_{2}$ production. The Warburg respirometer technique has made such determinations possible, but only rather small samples of soil can be used in such a case. SwabY and PAssey (4) have described a macro-respirometer for studies in soil microbiology. This apparatus, however, does not correct fluctuations in temperature and barometric pressure. In a preliminary note WIERINGA and KERKHoF (6) recommended the use of a macro-respirometer which counteracts these fluctuations. At the moment $I$ am using a respirometer of a more complicated design. In a container A (Fig. 2) of about $700 \mathrm{ml}$ is placed a gauze basket 
with a capacity of $100 \mathrm{~g}$ of soil. On the top of this respirometer (Fig. 3) are fixed 2 pairs of glass bulbs containing alkali. One set of bulbs is connected to the top of the respirometer and the other has a tube extending to the bottom. The air in the respirometer is sucked up and down via the bulbs by

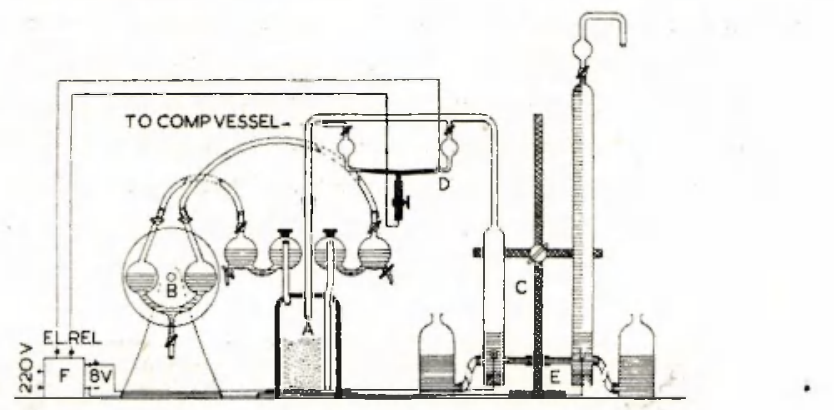

Fig. 2 Respiration assembly for soll samples (see text).

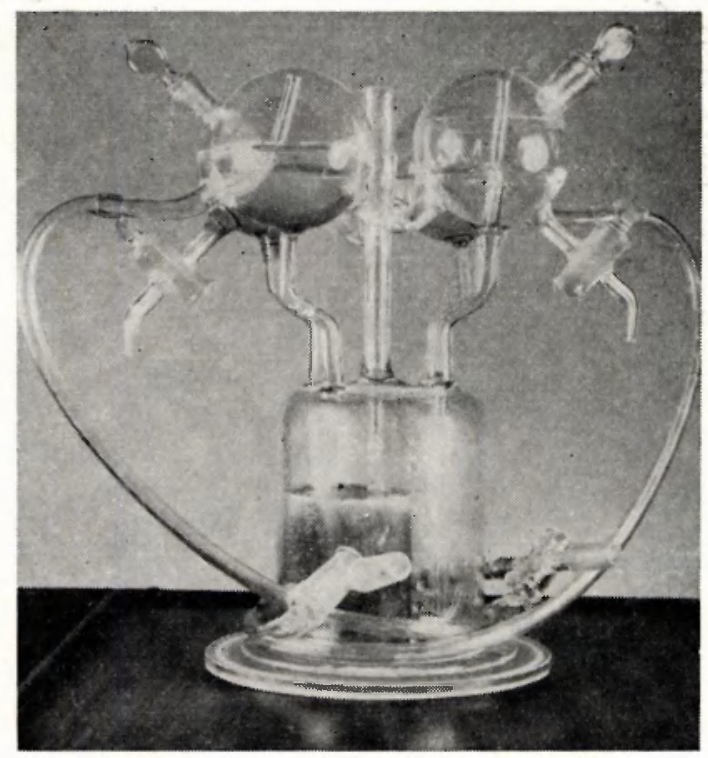

Fig. 3 Respirator for soil samples.

means of a swing $B$ with a water balance. A good contact of the air in the respirometer with the alkali is guaranteed. The top of the respirometer is connected to the oxygen-producing side of an electrolysis apparatus $\mathrm{C}$ which is provided with a side tube D. A capillary part of this tube is partly filled with mercury, one of the levels of which is brought between two electrodes. As soon as vacuum is formed in the respirometer an electric circuit is closed and the electrolysis apparatus begins to function. Oxygen is supplied to the system to make up the original pressure. The hydrogen produced by electrolysis is measured, and from this measurement it is possible to calculate the amount of oxygen taken up. To correct automatically fluctuations in tem- 
perature and barometric pressure, the tube connecting the oxygen and the hydrogen side of the electrolysis apparatus is provided with a tight asbestos fibre plug $\mathrm{E}$ soaked in the hydrolysis solution. This prevents water from flowing from the oxygen side to the hydrogen side of the electrolysis apparatus. The free end of the capillary tube $D$ is connected to a compensation vessel of the same capacity as the respirometer. In $\mathrm{F}$ are built in a rectifier producing a direct current of $8 \mathrm{~V}$ and an electronic relay controlling the direct current source. The respirometer system is entirely sealed off from the atmosphere and fluctuations in temperature and barometric pressure are automatically corrected. The readings of measured hydrogen should however be corrected for temperature and barometric pressure.

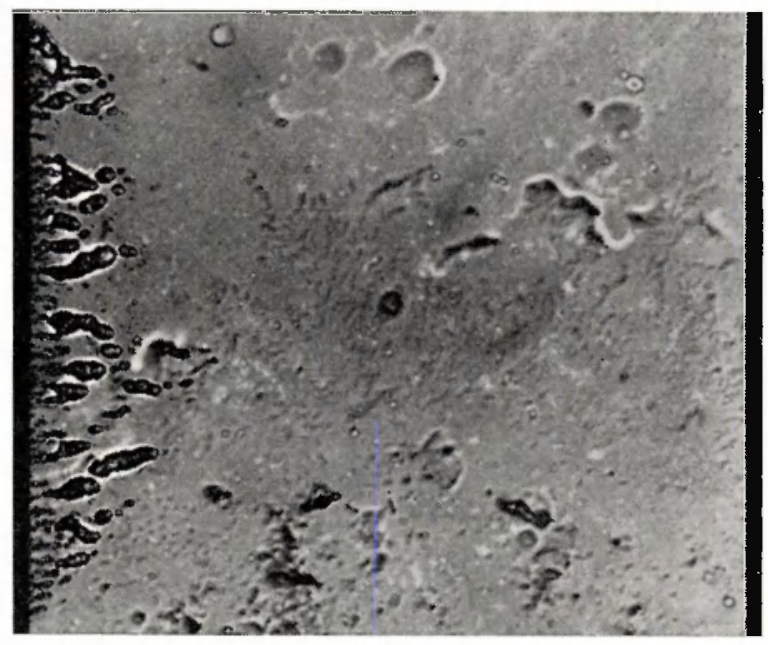

Fig. 4 Devfiofoment of Bacteria in the Cholodny soll chamber.

In experiments on production of $\mathrm{CO}_{2}$ and on respiration a peak is found at the start of the experiment. In my opinion this high respiratory activity is caused by abnormal conditions brought about by the preparation of the soil sample, e.g. screening and regulating the water contents. From the soil chamber method proposed by ChoLodNY (1) we learn that soil brought from the field to the laboratory may change considerably as regards the number of bacteria. Enormous masses of bacteria, both rods and cocci, as well as fungi may develop in the aqueous zone surrounding the soil particles (Fig. 4). I have not sufficient experience to know to what extent this depends on the biological possibilities of different soil types. In acid soils fungi do develop well under these conditions. It is certain, however, that in most samples brought to the laboratory bacterial numbers may alter greatly in two days. Thus it will be necessary to standardize the conditions under which soil samples are prepared.

To my knowledge, in all methods in which soil microbes and their activities are studied after the soil sample has been taken, we should take into account the disturbance caused by handling the soil sample. In the enzymatic 
method introduced by Hofmann (2), however, an attempt is made to study the microbial activity as it is present in the soil in its natural condition.

Hofmann studied the enzyme system present in the soil. He stated that in our soils enzymes are present in such quantities that they can be demonstrated by the methods used in enzyme chemistry. The soil is air-dried and screened. To 10 grams of soil thus treated there is added $1 \mathrm{ml}$ of toluene in order to prevent further bacterial growth. A phosphate buffer and the substrate are added afterwards and the dissimilation of the substrate is examined.

In one of his experiments he added to $10 \mathrm{~g}$ of soil $10 \mathrm{ml}$ of a phosphate buffer of $\mathrm{pH} 6.7$ and $10 \mathrm{ml}$ of a $10 \%$ urea solution, these being thoroughly mixed. The mixture was incubated at $37^{\circ} \mathrm{C}$. After $47 \mathrm{hrs}$. the mixture was filled up to $150 \mathrm{ml}$ with water of $37^{\circ} \mathrm{C}$. This mixture was shaken and left at $37^{\circ} \mathrm{C}$ for one more hour. The ammonia produced by urease activity was then distilled off in vacuo after the addition of NaOH. Hofmann and Schmm found significant differences between acid and neutral soils and samples taken at varying depths; grassland was more active than arable soil.

In this type of experiment growth of bacteria after the sampling is prevented if the air-drying is carried out immediately.

In standardizing our methods of determining the biological activity of the soil we first have to look to methods that are simple and of general importance. It is to be hoped that the use of these methods will provide us with sufficient information as to point the way to more detailed research.

Such methods, in addition to those concerning the physico-chemical properties of the soil (viz. soil texture, water capacity, organic matter, $\mathrm{pH}$, buffering capacity) already standardized by soil chemists, are methods of soil sampling, standardizing of the conditioning of the soil sample, methods of determining the mineralization capacity of the soil (respiration), and standardizing dilution methods, so as to enable the results to be treated mathematically ("Don't strain at a gnat and swallow a camel!")

So long as we are unable to standardize the soil sample and its preservation we shall get nowhere in standardizing the methods of biological examination of soil. In my opinion the microscopic examination of the soil in fluorescent light, introduced by Augier and РосноN, is the best method of verifying the effect of the preservation of the soil sample.

\section{REFERENCES}

1 Cholodny, N.: Arch. f. Mikrobiologie, 1 (1930) 620.

2 Hofmann, Ed. and G. Hofmann : Naturw. 40 (1953) 511.

3 Petersen, E. J.: Tidsskrift f. Planteavl, 32 (1926).

4 Swaby, R. J. and B. I. Passey : Austr. J. Agr. Res. (1953).

5 Waksman, S. A. : Principles of Soil Microbiology.

6 Wieringa, K. T. and F. M. Kerkhof Mogot : Plant and Soil 8 (1957) 395.

7 Winogradsky, S. : C.R. Ac. Sci. 178 (1924); id. Chimie et Ind. 2 (1924); id. 1er Congr. Int. du Sol, Rome 1924 ; id. Microbiol. du Sol. 